\title{
A REMARK ON THE EXISTENCE OF GLOBAL $B V$ SOLUTIONS FOR A NONLINEAR HYPERBOLIC WAVE EQUATION
}

\author{
BY \\ JOÃO-PAULO DIAS AND MÁRIO FIGUEIRA \\ CMAF/Univ. Lisboa, Av. Prof. Gama Pinto, 2, 1649-003 Lisboa, Portugal
}

Abstract. By means of a suitable change of variables we obtain, by application of a general result by Dafermos and Hsiao, cf. [2], an existence theorem in $L^{\infty} \cap B V_{\text {loc }}$ of a weak solution of the system corresponding to the quasilinear hyperbolic equation

$$
\phi_{t t}-p^{\prime}\left(\phi_{x}\right) \phi_{x x}+\phi_{t}+F(\phi)=0 \quad \text { in } \mathbb{R} \times[0,+\infty[,
$$

for small initial data in $B V$. This theorem is a partial extension of Dafermos's result for the case with $F(\phi) \equiv 0$, proved in [1].

1. The auxiliary system. Let us consider the following Cauchy problem:

$$
\begin{array}{ll}
\bar{\phi}_{t t}-p^{\prime}\left(\bar{\phi}_{x}\right) \bar{\phi}_{x x}+\bar{\phi}_{t}+\bar{F}(\bar{\phi})=0, & (x, t) \in \mathbb{R} \times[0,+\infty[, \\
\bar{\phi}(x, 0)=\bar{\phi}_{0}(x), \quad \bar{\phi}_{t}(x, 0)=\bar{v}_{0}(x), \quad x \in \mathbb{R},
\end{array}
$$

where $p$ is a given smooth function such that $p^{\prime}(\xi)>0, \forall \xi \in \mathbb{R}$, and $\bar{F}: \mathbb{R} \rightarrow \mathbb{R}$ is a smooth function verifying $\bar{F}(0)=0$. We assume, to simplify, the condition $p^{\prime}(0)=1$. Putting $u=\phi_{x}, v=\phi_{t}$ we can write (1.1), (1.2) as a Cauchy problem for a hyperbolic system:

$$
\begin{gathered}
\left\{\begin{array}{l}
\bar{\phi}_{t}=\bar{v} \\
\bar{u}_{t}=\bar{v}_{x} \\
\bar{v}_{t}-p^{\prime}(\bar{u}) \bar{u}_{x}+\bar{v}+\bar{F}(\bar{\phi})=0
\end{array} \quad(x, t) \in \mathbb{R} \times[0,+\infty[,\right. \\
\bar{\phi}(x, 0)=\bar{\phi}_{0}(x), \quad \bar{u}(x, 0)=\bar{u}_{0}(x)=\bar{\phi}_{0 x}(x), \quad \bar{v}(x, 0)=\bar{v}_{0}(x), \quad x \in \mathbb{R} .
\end{gathered}
$$

For technical reasons, if we choose $k>1$, we can, by putting $u(x, t)=\bar{u}(k x, k t), v(x, t)=$ $\bar{v}(k x, k t), \phi(x, t)=\frac{1}{k} \bar{\phi}(k x, k t)$, replace $(1.3),(1.4)$ by

$$
\left\{\begin{array}{l}
\phi_{t}=v \\
u_{t}=v_{x} \\
v_{t}-p^{\prime}(u) u_{x}+k v+F(\phi)=0
\end{array} \quad(x, t) \in \mathbb{R} \times[0,+\infty[,\right.
$$

Received March 14, 2000.

2000 Mathematics Subject Classification. Primary 35L70. 
where $F(\phi)=k \bar{F}(k \phi)$,

$$
\begin{aligned}
& \phi(x, 0)=\phi_{0}(x)=\frac{1}{k} \bar{\phi}_{0}(k x), \\
& u(x, 0)=u_{0}(x)=\bar{u}_{0}(k x)=\phi_{0 x}(x), \\
& v(x, 0)=v_{0}(x)=\bar{v}_{0}(k x), \quad x \in \mathbb{R} .
\end{aligned}
$$

For $m \in[1,+\infty[$, let us introduce

$$
\begin{aligned}
& a=a_{m}=\left(m^{2}-2 m+2\right)^{-1 / 2}, \\
& b=b_{m}=\left(m^{2}+2 m+2\right)^{-1 / 2} \\
& c_{m}=2-\left(\frac{a}{b}+\frac{b}{a}\right) / 2 \\
& k_{m}=2 c_{m}^{-1}(a(m-1)+b(m+1)) \\
& f_{m}=c_{m}^{2}\left[2\left(\frac{1}{a}+\frac{1}{b}\right)(a(m-1)+b(m+1))\right]^{-1}
\end{aligned}
$$

(notice that $c_{1}>0$ and $k_{1}>1$ ) and assume

$$
\left|\bar{F}^{\prime}(0)\right|<f_{1}
$$

We fix $m>1$ such that $\left|\bar{F}^{\prime}(0)\right|<f_{m}, c_{m}>0$, and $k_{m}>1$ and we put $k=k_{m}$. We obtain

$$
\begin{aligned}
& \left(2-\left(\frac{a}{b}+\frac{b}{a}\right) / 2\right) k-\left(\frac{1}{2 a}+\frac{1}{2 b}\right)\left|F^{\prime}(0)\right|-a(m-1)-b(m+1) \\
& =c_{m} k_{m}-\left(\frac{1}{2 a}+\frac{1}{2 b}\right) k_{m}^{2}\left|\bar{F}^{\prime}(0)\right|-a(m-1)-b(m+1)>0 .
\end{aligned}
$$

Now, we consider the following nonsingular linear transformation $(\phi, u, v) \rightarrow(\phi, u, w)$, where $w=v+m u+k \phi, m$ and $k$ as above. The Cauchy problem $\left(1.3^{\prime}\right),\left(1.4^{\prime}\right)$ takes the form

$$
\left\{\begin{array}{l}
\phi_{t}=w-m u-k \phi \\
u_{t}+k \phi_{x}+m u_{x}-w_{x}=0 \quad(x, t) \in \mathbb{R} \times[0,+\infty[ \\
w_{t}+m k \phi_{x}+\left(m^{2}-p^{\prime}(u)\right) u_{x}-m w_{x}+F(\phi)=0 \\
\phi(x, 0)=\phi_{0}(x) \\
u(x, 0)=u_{0}(x)=\phi_{0 x}(x), \\
w(x, 0)=w_{0}(x)=v_{0}(x)+m u_{0}(x)+k \phi_{0}(x), \quad x \in \mathbb{R} .
\end{array}\right.
$$


Now we introduce the following auxiliary Cauchy problem in $(\phi, u, w)$,

$$
\left\{\begin{array}{l}
\phi_{t}+m \phi_{x}-w+k \phi=0 \\
u_{t}+m u_{x}-w_{x}+k u=0 \\
w_{t}+\left(m^{2}-p^{\prime}(u)\right) u_{x}-m w_{x}+m k u+F(\phi)=0
\end{array} \quad \text { in } \mathbb{R} \times[0,+\infty[,\right.
$$

with the initial data $\left(1.4^{\prime \prime}\right)$.

If $(\phi, u, w)$ is a $C^{1}$ solution of $\left(1.3^{\prime \prime \prime}\right),\left(1.4^{\prime \prime}\right)$ we easily derive, for $\varphi \in C_{c}^{\infty}(\mathbb{R} \times[0,+\infty[)$,

$$
\begin{aligned}
-\int_{\mathbb{R}_{+}} \int_{\mathbb{R}} \phi \varphi_{x t} d x d t- & \int_{\mathbb{R}} \phi_{0} \varphi_{x}(\cdot, 0) d x-m \int_{\mathbb{R}_{+}} \int_{\mathbb{R}} \phi \varphi_{x x} d x d t \\
& -\int_{\mathbb{R}_{+}} \int_{\mathbb{R}} w \varphi_{x} d x d t+k \int_{\mathbb{R}_{+}} \int_{\mathbb{R}} \phi \varphi_{x} d x d t=0
\end{aligned}
$$

and

$$
\begin{aligned}
-\int_{\mathbb{R}_{+}} \int_{\mathbb{R}} u \varphi_{t} d x d t-\int_{\mathbb{R}} & u_{0} \varphi(\cdot, 0) d x-m \int_{\mathbb{R}_{+}} \int_{\mathbb{R}} u \varphi_{x} d x d t \\
& \quad+\int_{\mathbb{R}_{+}} \int_{\mathbb{R}} w \varphi_{x} d x d t+k \int_{\mathbb{R}_{+}} \int_{\mathbb{R}} u \varphi d x d t=0,
\end{aligned}
$$

and so, by addition, we obtain

$$
\int_{\mathbb{R}_{+}} \int_{\mathbb{R}} \phi\left[\varphi_{t}+m \varphi_{x}-k \varphi\right]_{x} d x d t+\int_{\mathbb{R}_{+}} \int_{\mathbb{R}} u\left[\varphi_{t}+m \varphi_{x}-k \varphi\right] d x d t=0 .
$$

Now, given $\psi \in \mathcal{D}(\mathbb{R} \times] 0,+\infty[)$, it is easy to find $\varphi \in C_{c}^{\infty}(\mathbb{R} \times[0,+\infty[)$ such that $\varphi_{t}+m \varphi_{x}-k \varphi=\psi$ : first, with $\psi_{1}=e^{-k t} \psi, \varphi_{1}=e^{-k \varphi} \varphi$, we reduce to $\varphi_{1 t}+m \varphi_{1 x}=\psi_{1}$. We put

$$
\varphi_{1}(m t+c, t)=\int_{0}^{t} \psi_{1}(m \tau+c, \tau) d \tau-\widetilde{\varphi}_{1}(c)
$$

where

$$
\widetilde{\varphi}_{1}(c)=\int_{0}^{+\infty} \psi_{1}(m t+c, t) d t, \quad \forall c \in \mathbb{R}
$$

Hence,

$$
\int_{\mathbb{R}_{+}} \int_{\mathbb{R}} \phi \psi_{x} d x d t+\int_{\mathbb{R}_{+}} \int_{\mathbb{R}} u \psi d x d t=0, \quad \forall \psi \in \mathcal{D}(\mathbb{R} \times] 0,+\infty[)
$$

We derive $u=\phi_{x}$. It is now easy to prove

Proposition 1.1. For given initial data $\left(\phi_{0}, u_{0}=\phi_{0 x}, w_{0}\right)$ in $C^{1}(\mathbb{R}) \cap W^{1, \infty}(\mathbb{R})$ the systems $\left(1.3^{\prime \prime}\right)$ and $\left(1.3^{\prime \prime \prime}\right)$ have the same (local in time) $C^{1}$ solutions. 
Given $\left(\phi_{0}, u_{0}, w_{0}\right)$ in $L^{\infty}(\mathbb{R})$, we say, as usually, that $(\phi, u, w) \in\left(L_{\text {loc }}^{\infty}\left(\mathbb{R} \times[0,+\infty[))^{3}\right.\right.$ is a weak (global) solution of the Cauchy problem $\left(1.3^{\prime \prime}\right),\left(1.4^{\prime \prime}\right)$ if we have

$$
\begin{gathered}
\int_{\mathbb{R}_{+}} \int_{\mathbb{R}} \phi \varphi_{t} d x d t+\int_{\mathbb{R}} \phi_{0} \varphi(\cdot, 0) d x+\int_{\mathbb{R}_{+}} \int_{\mathbb{R}}(w-m u-k \phi) \varphi d x d t \\
\quad+\int_{\mathbb{R}_{+}} \int_{\mathbb{R}} u \psi_{t} d x d t+\int_{\mathbb{R}} u_{0} \psi(\cdot, 0) d x+\int_{\mathbb{R}_{+}} \int_{\mathbb{R}}(k \phi+m u-w) \psi_{x} d x d t \\
\quad+\int_{\mathbb{R}_{+}} \int_{\mathbb{R}} w \theta_{t} d x d t+\int_{\mathbb{R}} w_{0} \theta(\cdot, 0) d x \\
\quad+\int_{\mathbb{R}_{+}} \int_{\mathbb{R}}\left[m^{2} u-p(u)-m w\right] \theta_{x} d x d t-\int_{\mathbb{R}_{+}} \int_{\mathbb{R}} F(\phi) \theta d x d t=0, \\
\forall \varphi, \psi, \theta \in C_{c}^{\infty}(\mathbb{R} \times[0,+\infty[),
\end{gathered}
$$

and a similar definition for the Cauchy problem $\left(1.3^{\prime \prime \prime}\right),\left(1.4^{\prime \prime}\right)$.

We can repeat the calculations made to prove Proposition 1.1 for a $(\phi, u, w) \in$ $\left(L_{\text {loc }}^{\infty}\left(\mathbb{R} \times[0,+\infty[))^{3}\right.\right.$ weak solution of $\left(1.3^{\prime \prime \prime}\right),\left(1.4^{\prime \prime}\right)$ and we obtain $\phi_{x}=u$ in $\mathcal{D}^{\prime}(\mathbb{R} \times] 0,+\infty[)$. We derive $\phi \in W_{\mathrm{loc}}^{1, \infty}(\mathbb{R} \times[0,+\infty[)$ and it is now easy to prove that $(\phi, u, w)$ is a weak solution of $\left(1.3^{\prime \prime}\right),\left(1.4^{\prime \prime}\right)$. The converse is also true, by similar considerations. Hence, we have

Proposition 1.2. For a given initial data $\left(\phi_{0}, u_{0}=\phi_{0 x}, w_{0}\right)$ in $L^{\infty}(\mathbb{R})$, the systems $\left(1.3^{\prime \prime}\right)$ and $\left(1.3^{\prime \prime \prime}\right)$ have the same weak solutions.

Now let $(\eta, q)$ be a pair of smooth convex entropy/entropy flux for the system $\left(1.3^{\prime \prime}\right)$ (cf. [4]).

$$
\begin{aligned}
& \left(\text { Example: } \quad \eta_{1}(\phi, u, w)=\frac{1}{2} \phi^{2}+\int_{0}^{u} p(\xi) d \xi+\frac{1}{2}(w-m u-k \phi)^{2},\right. \\
& \left.q_{1}(\phi, u, w)=-(w-m u-k \phi) p(u)\right) \text {. }
\end{aligned}
$$

A weak solution $(\phi, u, w)$ of $\left(1.3^{\prime \prime}\right),\left(1.4^{\prime \prime}\right)$ is called an entropy weak solution if, in $\mathcal{D}^{\prime}(\mathbb{R} \times] 0,+\infty[)$,

$$
\eta(\phi, u, w)_{t}+q(\phi, u, w)_{x}+\nabla \eta \cdot(-w+m u+k \phi, 0, F(\phi)) \leq 0
$$

for all pairs $(\eta, q), \eta$ convex.

The system $\left(1.3^{\prime \prime \prime}\right)$ admits the entropy/entropy flux pair $\left(\widetilde{\eta}_{1}, \widetilde{q}_{1}\right), \widetilde{\eta}_{1}$ strictly convex, defined by

$$
\begin{aligned}
& \widetilde{\eta}_{1}(\phi, u, w)=\frac{1}{2} \phi^{2}+\int_{0}^{u} p(\xi) d \xi+\frac{1}{2}(w-m u)^{2} \\
& \widetilde{q}_{1}(\phi, u, w)=\frac{1}{2} m \phi^{2}-(w-m u) p(u) .
\end{aligned}
$$

If $(\phi, u, w) \in\left(L_{\mathrm{loc}}^{\infty} \cap B V_{\mathrm{loc}}\right)^{3}$ is a weak solution of $\left(1.3^{\prime \prime}\right),\left(1.4^{\prime \prime}\right)$ with initial data in $B V(\mathbb{R})$, we can prove (with some tedious computations, taking in mind that $\phi_{x}=$ 
$u$, cf. Proposition 1.2, and applying the theorem in section 13.2 of [5] concerning the differentiation of the composition) that we have, in $\mathcal{D}^{\prime}(\mathbb{R} \times] 0,+\infty[)$,

$$
\begin{aligned}
\tilde{\eta}_{1}(\phi, u, w)_{t} & +\widetilde{q}_{1}(\phi, u, w)_{x}+\nabla \widetilde{\eta}_{1} \cdot(-w+k \phi, k u, m k u+F(\phi)) \\
= & \eta_{1}(\phi, u, w)_{t}+q_{1}(\phi, u, w)_{x}+\nabla \eta_{1} \cdot(-w+m u+k \phi, 0, F(\phi)) .
\end{aligned}
$$

Hence, by Proposition 1.2, we conclude

TheOREM 1.3. Assume that $\left(\phi_{0}, u_{0}=\phi_{0 x}, w_{0}\right) \in B V(\mathbb{R})^{3}$ and let $(\phi, u, w) \in\left(L_{\text {loc }}^{\infty} \cap\right.$ $B V_{\text {loc }}\left(\mathbb{R} \times[0,+\infty[))^{3}\right.$ be an entropy weak solution of $\left(1.3^{\prime \prime \prime}\right),\left(1.4^{\prime \prime}\right)$. Then, $(\phi, u, w)$ is also a weak solution of $\left(1.3^{\prime \prime}\right),\left(1.4^{\prime \prime}\right)$ verifying $(1.8)$ for the pair $\left(\eta_{1}, q_{1}\right)$ defined above.

2. Application of Theorem 2 in [2]. Now, in order to apply to the Cauchy problem $\left(1.3^{\prime \prime \prime}\right),\left(1.4^{\prime \prime}\right)$ Theorem 2 in [2], we give initial data $\left(\phi_{0}, u_{0}=\phi_{0 x}, w_{0}\right)$ in $B V(\mathbb{R})$. The system $\left(1.3^{\prime \prime \prime}\right)$ can be written as follows (recall that $p^{\prime}(0)=1$ and $m>1$ ):

$$
\frac{\partial}{\partial t}\left(\begin{array}{c}
\phi \\
u \\
w
\end{array}\right)+A(u) \frac{\partial}{\partial x}\left(\begin{array}{c}
\phi \\
u \\
w
\end{array}\right)+g(\phi, u, w)=0
$$

where

$$
A(u)=\left(\begin{array}{ccc}
m & 0 & 0 \\
0 & m & -1 \\
0 & m^{2}-p^{\prime}(u) & -m
\end{array}\right), \quad g(\phi, u, w)=\left(\begin{array}{c}
-w+k \phi \\
k u \\
m k u+F(\phi)
\end{array}\right) .
$$

The eigenvalues of $A(u)$ are $\left(m, \sqrt{p^{\prime}(u)},-\sqrt{p^{\prime}(u)}\right)$. The matrix of the corresponding (independent) normalized right eigenvectors for $u=0$ is

$$
B=\left(\begin{array}{ccc}
1 & 0 & 0 \\
0 & a & b \\
0 & a(m-1) & b(m+1)
\end{array}\right) \quad \text { and } \nabla g(0,0,0)=\left(\begin{array}{ccc}
k & 0 & -1 \\
0 & k & 0 \\
F^{\prime}(0) & m k & 0
\end{array}\right) \text {, }
$$

where $a=\left(m^{2}-2 m+2\right)^{-1 / 2}$ and $b=\left(m^{2}+2 m+2\right)^{-1 / 2}$.

Hence, $R=\left\{r_{i j}\right\}=B^{-1} \nabla g(0,0,0) B$ is given by

$$
R=\left(\begin{array}{ccc}
k & a(-m+1) & b(-m-1) \\
-\frac{1}{2 a} F^{\prime}(0) & \frac{k}{2} & \frac{b}{a} \frac{k}{2} \\
\frac{1}{2 b} F^{\prime}(0) & \frac{a}{b} \frac{k}{2} & \frac{k}{2}
\end{array}\right)
$$

and verifies

$$
\begin{aligned}
\sum_{i} r_{i i}-\sum_{i \neq j}\left|r_{i j}\right|= & \left(2-\left(\frac{a}{b}+\frac{b}{a}\right) / 2\right) k-\left(\frac{1}{2 a}+\frac{1}{2 b}\right)\left|F^{\prime}(0)\right| \\
& -a(m-1)-b(m+1)>0 \quad \text { by }(1.6)
\end{aligned}
$$

and so $R$ is diagonal dominant.

By applying Theorem 2 in [2] we derive 
Theorem 2.1. Let us assume (1.5). Then, there exist two positive constants $a_{0}, b_{0}>0$ such that, if

$$
\left(\phi_{0}, u_{0}=\phi_{0 x}, w_{0}\right) \in(B V(\mathbb{R}))^{3}
$$

and

$$
\left\|\left(\phi_{0}, u_{0}, w_{0}\right)\right\|_{L^{\infty}(\mathbb{R})} \leq a_{0}, \quad T V_{x}\left(\phi_{0}, u_{0}, w_{0}\right) \leq b_{0},
$$

then there exists a weak entropy solution $(\phi, u, w) \in\left(L^{\infty} \cap B V_{\text {loc }}\left(\mathbb{R} \times[0,+\infty[))^{3}\right.\right.$ of $\left(1.3^{\prime \prime \prime}\right),\left(1.4^{\prime \prime}\right)$. Moreover $(\phi(\cdot, t), u(\cdot, t), w(\cdot, t)) \in(B V(\mathbb{R}))^{3}$ for each $t \geq 0$, with a uniformly bounded (in $t$ ) total variation $T V_{x}$.

Hence, by Theorem 1.3, we can derive a similar result for the Cauchy problem (1.3), (1.4) if we replace the general entropy condition (1.8) by the following particular one:

$$
\bar{\eta}(\bar{\phi}, \bar{u}, \bar{v})_{t}+\bar{q}(\bar{\phi}, \bar{u}, \bar{v})_{x}+\nabla \bar{\eta} \cdot(-\bar{v}, 0, \bar{v}+\bar{F}(\bar{\phi})) \leq 0 \quad \text { in } \quad \mathcal{D}^{\prime}(\mathbb{R} \times] 0,+\infty[)
$$

where

$$
(\bar{\eta}, \bar{q})=\left(\frac{1}{2} \bar{\phi}^{2}+\int_{0}^{\bar{u}} p(\xi) d \xi+\frac{1}{2} \bar{v}^{2},-\bar{v} p(\bar{u})\right) .
$$

See [1] for the case with $\bar{F} \equiv 0$ and [3] for related results.

Acknowledgments. This work was supported by FCT, PRAXIS XXI, FEDER and Project PRAXIS/2/2.1/MAT/125/94.

\section{REFERENCES}

[1] C. M. Dafermos, A system of hyperbolic conservation laws with frictional damping, ZAMP 46, S294-S307 (1995)

[2] C. M. Dafermos and L. Hsiao, Hyperbolic systems of balance laws with inhomogeneity and dissipation, Indiana Univ. Math. J. 31, 471-491 (1982)

[3] T.-P. Liu, Quasilinear hyperbolic systems, Commun. Math. Phys. 68, 141-172 (1979)

[4] J. A. Smoller, Shock Waves and Reaction-Diffusion Equations, Springer-Verlag, New York, 1983

[5] A. I. Vol'pert, The spaces BV and quasilinear equations, Math. USSR Sbornik 2, 225-267 (1967) 